\title{
Markers of endothelial cell activation in suspected late onset neonatal sepsis in Surinamese newborns: a pilot study
}

\author{
Niek B. Achten ${ }^{1,2}$, Matijs van Meurs ${ }^{3,4,5}$, Rianne M. Jongman ${ }^{3,4,5}$, Amadu Juliana ${ }^{1}$, Grietje Molema ${ }^{3}$, \\ Frans B. Plötz ${ }^{2}$, Rens Zonneveld ${ }^{1,3}$ \\ ${ }^{1}$ Academic Pediatric Center Suriname, Academic Hospital Paramaribo, Paramaribo, Suriname; ${ }^{2}$ Department of Pediatrics, Tergooi Hospitals, \\ Blaricum, The Netherlands; ${ }^{3}$ Department of Pathology and Medical Biology, ${ }^{4}$ Department of Critical Care, ${ }^{5}$ Department of Anesthesiology, \\ University Medical Center Groningen, University of Groningen, Groningen, The Netherlands \\ Contributions: (I) Conception and design: R Zonneveld, FB Plotz; (II) Administrative support: All authors; (III) Provision of study materials or \\ patients: A Juliana; (IV) Collection and assembly of data: R Zonneveld, NB Achten, A Juliana; (V) Data analysis and interpretation: NB Achten, RM \\ Jongman, R Zonneveld; (VI) Manuscript writing: All authors; (VII) Final approval of manuscript: All authors. \\ Correspondence to: Niek Achten, MD. Department of Pediatrics, Tergooi Hospitals, Rijksstraatweg 9, Blaricum, The Netherlands. \\ Email: niek.achten+tp@gmail.com.
}

\begin{abstract}
Background: Serum levels of markers of endothelial cell activation are associated with bacteremia and mortality in sepsis in adults, children, and newborns with early onset sepsis. We hypothesize that levels of these markers are associated with these outcomes in hospitalized newborns with suspected late onset neonatal sepsis (LONS).

Methods: In this prospective cohort study, newborns admitted to the tertiary neonatal care facility of Suriname were included upon clinical suspicion of LONS and before start of antibiotic treatment, between April 1, 2015 and May 31, 2016. Serum concentrations of angiopoietin-1, angiopoietin-2, and soluble isoforms of P-selectin, E-selectin, vascular cell adhesion molecule-1 (sVCAM-1), intercellular adhesion molecule-1 (sICAM-1), platelet and endothelial cell adhesion molecule-1 (sPECAM-1), matrix metalloproteinase-9 (MMP-9), neutrophil elastase, and tissue-inhibitor of metalloproteinases-1 (TIMP-1) were measured.

Results: Twenty-thee newborns were included. Baseline characteristics were similar between newborns with and without bacteremia and between non-survivors and survivors. Only soluble E-selectin (sE-selectin) was higher in newborns with bacteremia versus non-bacteremia $(\mathrm{P}=0.04)$ and lower in non-survivors $(\mathrm{P}=0.04)$. No conclusions could be made for sVCAM-1 due to high serum concentrations.

Conclusions: In conclusion, the data from this pilot study indicate that serum levels of markers of endothelial cell activation are poorly associated with bacteremia and mortality.
\end{abstract}

Keywords: Endothelial cell; newborn; sepsis

Submitted Jul 16, 2019. Accepted for publication Nov 13, 2019.

doi: $10.21037 /$ tp.2019.11.03

View this article at: http://dx.doi.org/10.21037/tp.2019.11.03

\section{Introduction}

A key feature of sepsis pathophysiology is endothelial cell activation (1). During sepsis, a shift in balance in vascular endothelial Tie2 receptor ligands angiopoietin (Ang)-1 and Ang-2 affects endothelial integrity, causing microvascular permeability $(2,3)$. Occurrence of this shift is followed by increased expression of endothelial cell adhesion molecules (CAMs) that orchestrate interactions between leukocytes and microvascular endothelial cells. During sepsis, soluble isoforms of CAMs (sCAMs) are released into the bloodstream by sheddases, such as matrix metalloproteinase-9 (MMP-9), tissue inhibitor metalloproteinase-1 (TIMP-1) and neutrophil elastase (NE) (4,5). 
Clinical studies $(5,6)$ confirm that increased levels of Ang-2 and Ang-2/Ang-1 ratio upon admission to the emergency room or intensive care unit are associated with bacteremia and/or mortality in adults (7), children (8), and young infants (9). In addition, it has been suggested that the angiopoietins may also be a potential marker for clinical use in newborns (10). Recently, our group indeed demonstrated in newborns with suspected early onset sepsis (EOS), defined as onset of sepsis within 72 hours after birth, that decreased Ang-1 serum levels, increased serum Ang-2 level, and an increased Ang-2/Ang-1 ratio were associated with bacteremia (11). However, no differences were found in serum levels of sCAMs and their sheddases between bacteremia and non-bacteremia in EOS (12). For late onset neonatal sepsis (LONS), defined as onset of sepsis after 72 hours after birth, in newborns admitted to neonatal wards data on levels of these markers are absent, but may be clinically relevant.

For this pilot study, we evaluated levels of a panel of angiopoietins and sCAMs and their sheddases in Surinamese newborns with suspected LONS, admitted to the only tertiary neonatal care facility in Suriname. Based on the available literature in adults and EOS, we hypothesized that lower Ang-1, higher Ang-2, and higher sCAM and sheddase levels associate with bacteremia or mortality in suspected LONS.

\section{Methods}

\section{Study design and subjects}

This was an observational prospective cohort study conducted amongst newborns admitted to the tertiary neonatal care facility of the Academic Hospital Paramaribo, Paramaribo, Suriname (13). Between April 1, 2015 and May 31, 2016, newborns were included with suspected LONS during postnatal hospital admission up to 90 days of age. LONS was suspected upon presence of clinical symptoms of LONS in newborns older than 72 hours of age for which antibiotics were started after blood culturing by experienced treating pediatricians. Newborns with a gestational age below 27 weeks were not actively treated and therefore not included (13). Excluded were newborns of whom no or limited clinical data or serum was available, and newborns born to HIV positive mothers. Primary outcome was bacteremia and secondary outcome was mortality, defined as in-house mortality within 28 days after inclusion.

\section{Clinical protocol and data collection}

Local protocol for suspected LONS included sampling of serum for clinical infection parameter C-reactive protein (CRP), and EDTA-plasma for whole blood analysis of cell counts and hematological parameters. All samples and blood cultures were obtained via one venipuncture with an intravenous catheter prior to start of intravenous antibiotics. Standard intravenous antibiotic regime consisted of amoxicillin/clavulate acid (i.e., 50/5$100 / 10 \mathrm{mg} / \mathrm{kg} / 24 \mathrm{~h}$ ) with amikacin (i.e., $15-18 \mathrm{mg} / \mathrm{kg} / 24$ $48 \mathrm{~h}$ ). After inclusion, data regarding maternal characteristics and perinatal parameters, clinical symptoms, laboratory results, and outcomes was collected from the paper medical charts and discharge letters, where available.

\section{Sample collection and analysis}

Residual serum was harvested at the local clinical laboratory as described before (11), stored immediately at $-80{ }^{\circ} \mathrm{C}$, and was transported on dry ice from Suriname to The Netherlands. Serum samples were aliquoted and analyzed as described before $(11,12)$. In brief, aliquots of the same sample were used for measurement of Ang-1 and Ang2 with the Human Luminex Screening Assay (LXSAH, R\&D Systems, Abingdon, UK), measurement of soluble $\mathrm{P}$-selectin (sP-selectin), soluble E-selectin (sE-selectin), sVCAM-1, soluble intercellular adhesion molecule-1 (sICAM-1), and soluble platelet and endothelial cell adhesion molecule-1 (sPECAM-1) with the Human Magnetic Bead Adhesion 6-plex panel performance assay (LHC0016M, Life Technologies, Bleiswijk, The Netherlands) and measurement of MMP-9 (Quantikine DMP900, R\&D systems), TIMP-1 (Quantikine DTM100, R\&D systems), and NE (HK319, Hycult Biotech, Uden, The Netherlands) with ELISA, all according to the manufacturers' instructions. For each molecule, a standard curve was established to determine levels in neonatal serum. Levels above the linear part [i.e., for $\mathrm{sE}$-selectin $\mathrm{n}=4, \mathrm{MMP}$ $9 \mathrm{n}=1$, and for $\mathrm{NE} \mathrm{n}=4$ samples in this study, respectively] of this standard curve are reported as the highest value of the standard curve. Inter-assay coefficients of variation were determined and a maximum of $20 \%$ was accepted.

\section{Statistical analysis}

Baseline characteristics and distributions of clinical 
symptoms were compared using chi-square to test for statistical significance. All biomarker data were presented as median with interquartile range (IQR) and compared using Mann-Whitney $\mathrm{U}$ tests due to non-normality in data distributions. P values $<0.05$ were considered statistically significant. All statistical analysis was performed using $\mathrm{R}$ version 3.5.0 (R Foundation, Vienna, Austria), and Prism version 7.0 (Graphpad Software Inc., San Diego, CA, USA).

\section{Ethics statement}

The Surinamese Medical-Ethical Board approved the study (VG-021-14A) and an amendment (VG-02114A-2015_2016) for inclusion of newborns with a GA below 32 weeks and/or birth weight below 1,500 grams. The original protocol for the biomarker study amongst Surinamese newborns was registered on clinicaltrials.gov (Trial registration: NCT02486783, registered 27/6/2015). Written informed consent for the use of serum and clinical information was obtained from at least one parent. The study was conducted in accordance with the Helsinki Declaration.

\section{Results}

\section{Baseline characteristics}

Thirty-one newborns were eligible of whom 23 were included (Table 1). Excluded were one newborn with maternal HIV, and 7 newborns without confirmed blood culture result. Most newborns were born premature with a gestational age below 37 weeks $(n=21,91 \%)$. Median birthweight was 1,280 (IQR 1,144) grams and median age at presentation was 9 (IQR 15) days. Nine (41\%) newborns were born with a caesarean section. Serum levels of Ang1, Ang-2, MMP-9, and TIMP-1 could be determined for all 23 newborns. However, serum levels of all sCAMs and $\mathrm{NE}$ could be determined for $\mathrm{n}=19(83 \%)$ newborns, due to insufficient availability of serum for all assays.

\section{Bacteremia and markers of endothelial cell activation}

Seven $(30 \%)$ newborns had bacteremia with gram-negative pathogens, namely Klebsiella pneumoniae $(\mathrm{n}=3)$, Pantoea species $(\mathrm{n}=1)$, Enterobacter cloacae $(\mathrm{n}=1)$, Stenotrophomonas maltophilia $(\mathrm{n}=1)$, and one co-infection of Escherichia coli with Serratia marcescens occurred. Three newborns with a blood culture with a coagulase-negative Staphylococcus (CNS) were considered contamination and therefore classified as non-bacteremia. Only serum levels of $\mathrm{sE-selectin} \mathrm{were}$ different between newborns with bacteremia and newborns without bacteremia (Figure 1). Levels for sVCAM-1 were high in all serum samples and above the linear part of the standard curve in the large majority $(n=13)$ of samples, making meaningful interpretations impossible. Limited serum sample availability precluded further dilution and sVCAM-1 was removed from the analysis. No statistically significant differences were observed in serum levels of the other markers between newborns with and without bacteremia.

\section{Mortality and markers of endothelial cell activation}

Four $(17 \%)$ newborns died during admission, all within 2 weeks after the sampling date [median 5.5 (IQR 4.5) days]. Of these newborns, one newborn had a positive blood culture with Stenotrophomonas maltophilia. No significant differences in baseline characteristics were found between non-survivors and survivors (Table 1). Only sE-selectin levels were lower in non-survivors $(\mathrm{P}=0.04)$ (Figure 1). Levels of none of the other markers were different between non-survivors and survivors (Figure 1).

\section{Discussion}

In this pilot study, we investigated association of serum levels of markers of endothelial cell activation and specific sheddases with bacteremia and mortality in suspected late neonatal sepsis in admitted newborns. In contrast to our hypothesis, only higher levels of sE-selectin were associated with bacteremia and lower levels of sE-selectin with mortality. For sVCAM-1 no conclusions could be made. None of the other markers were different between groups.

The lack of a clear association of markers of endothelial cell activation with bacteremia and mortality in newborns with suspected LONS contrasts with earlier studies in children and adults (14-16). Both Pierce et al. and Wright et al. found higher Ang-2 levels and a higher Ang-2/Ang1 ratio to be associated with presence of sepsis, bacteremia and/or mortality in infants presenting at the emergency department or the pediatric intensive care unit $(9,17)$. Similar to these studies, our cohort consisted of critically ill patients as indicated by high rates of comorbidity, interventions, and mortality. However, subjects were often included upon admission, whereas our cohort consisted of subjects that were already admitted to the neonatal care 
Table 1 Demographic and clinical characteristics of the study group by bacteremia and mortality

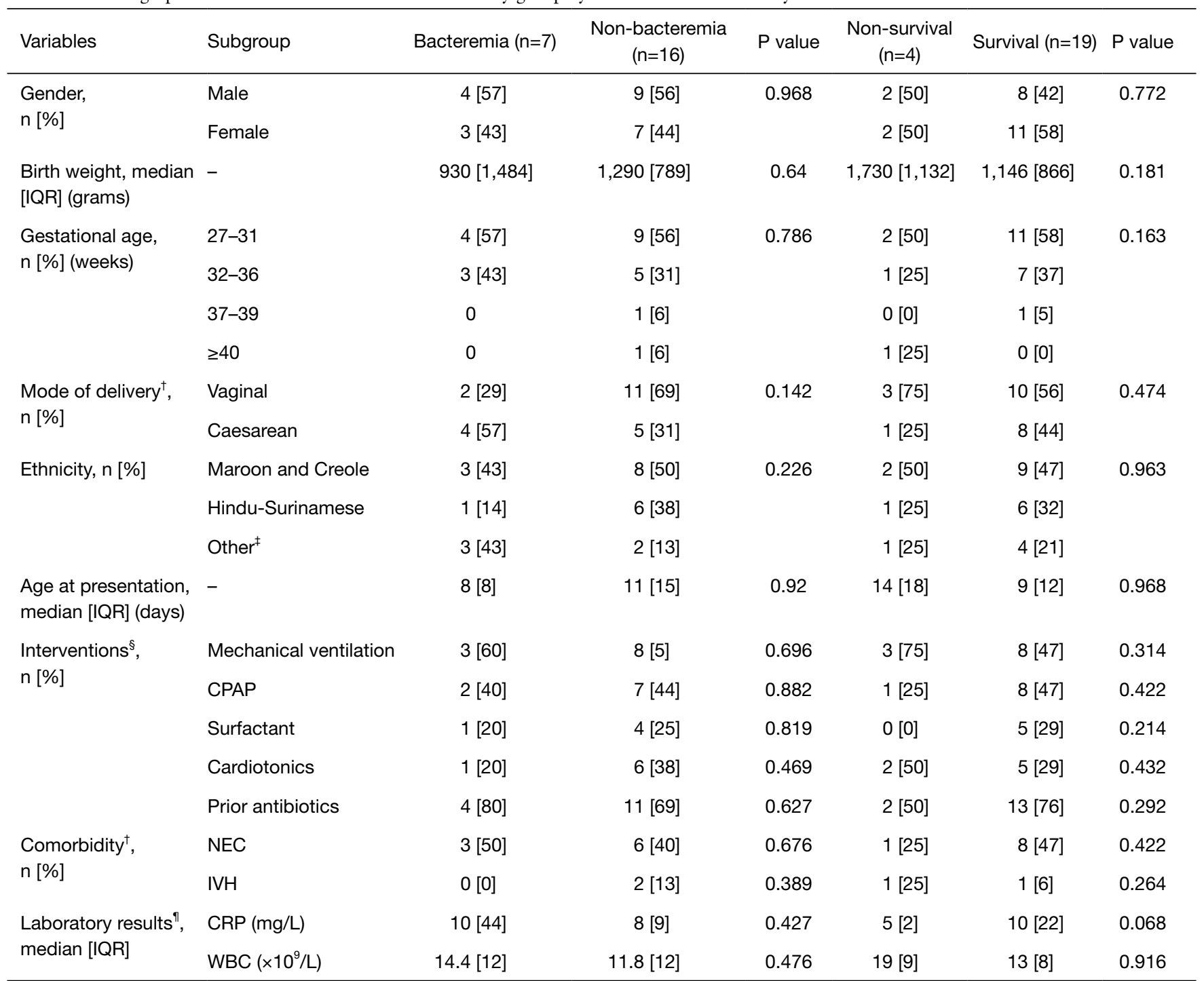

${ }^{\dagger}$, data on mode of delivery, NEC and IVH unavailable in $n=1, n=2$ and $n=3$ newborns, respectively; ${ }^{\ddagger}$, includes: Javanese, Chinese, Caucasian and Amerindian; ${ }^{\S}$, reported are interventions performed before inclusion and/or during late onset sepsis episode. Data unavailable of $n=2$ newborns; ", reported are levels at start of antibiotic treatment. Data on CRP and WBC unavailable in $n=2$ and $\mathrm{n}=3$ newborns, respectively. IQR, interquartile range; CPAP, continuous positive airway pressure; NEC, necrotizing enterocolitis; IVH, intraventricular hemorrhage; WBC, white blood cell count.

facility prior to sampling. In these newborns, common neonatal comorbidities may have affected endothelial cell activation, thereby limiting discriminatory potential of levels of its markers for LONS. For example, presence of necrotizing enterocolitis is associated with higher levels of Ang-2 in preterm infants (18). Hypothetically, this may lead to a shift in balance in Ang-1 and Ang-2 levels in favor of Ang-2 thereby saturating the endothelial Tie-2 receptor leading to high rates of receptor dephosphorylation $(2,3)$.
This may sensitize the endothelium for inflammatory reactivity, represented by increased CAM upregulation and expression and sCAM shedding into the vasculature $(4,19)$, before LONS is present. Inflammatory stimuli during LONS may then have no measurable effect on circulating serum levels of angiopoietins and sCAMs. The latter is supported by the fact that overall levels of the angiopoietins in the current study appear relatively high when compared to healthy controls in EOS and in children and adults 
A

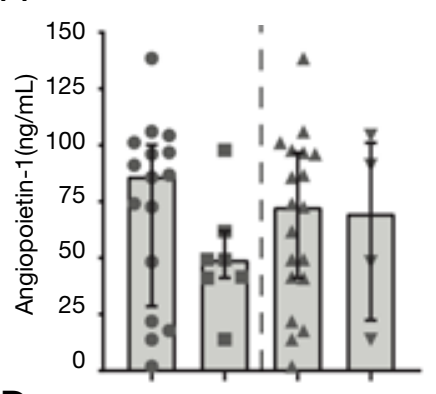

D

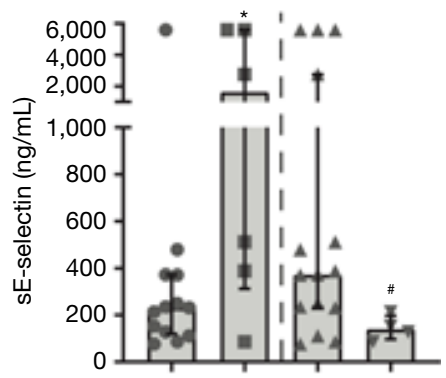

$\mathrm{H}$

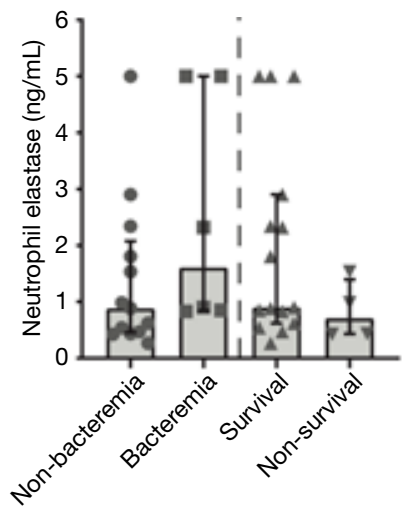

B

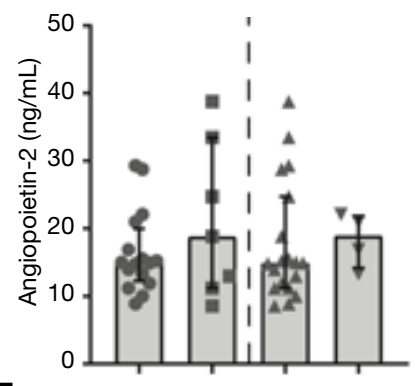

E

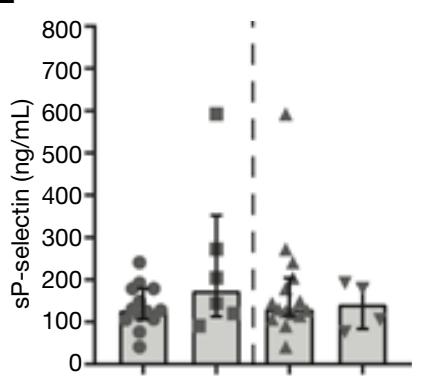

1

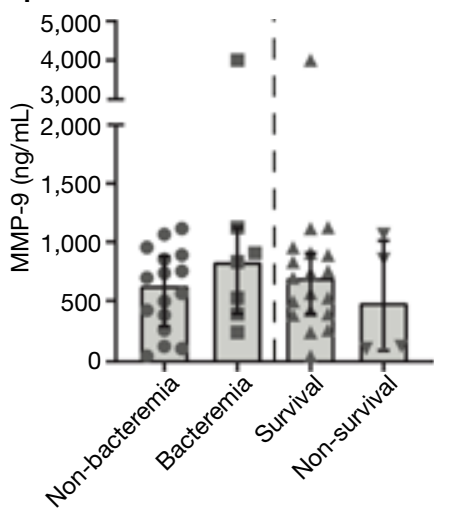

C
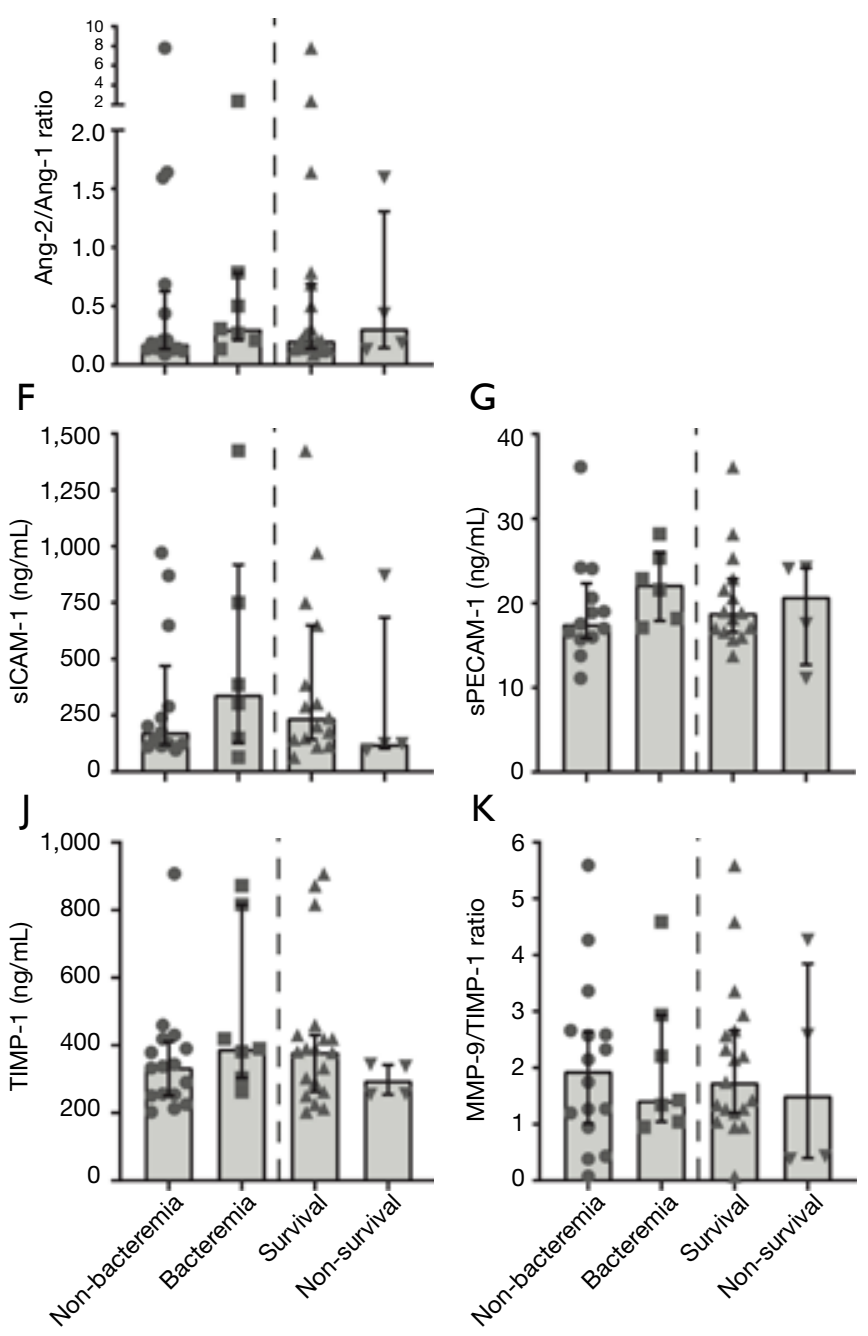

Figure 1 Serum levels of markers of endothelial cell activation in Surinamese newborns with suspected late onset neonatal sepsis grouped by the presence of bacteremia and mortality. (A) Angiopoietin (Ang)-1; (B) Ang-2; (C) Ang-2/Ang-1 ratio; (D) sE-selectin; (E) sP-selectin; (F) sICAM-1; (G) sPECAM-1; (H) neutrophil elastase; (I) MMP-9; (J) TIMP-1; (K) MMP-9/TIMP-1 ratio. Bars represent median values reported and error bars interquartile range. *, $\mathrm{P}<0.05$ between bacteremia and non-bacteremia; ${ }^{*}, \mathrm{P}<0.05$ between non-survival and survival. All analyses performed with a Mann-Whitney $\mathrm{U}$ test. $\mathrm{P}<0.05$ was considered statistically significant. sE-selectin, soluble E-selectin; sPselectin, soluble P-selectin; sICAM-1, soluble intercellular adhesion molecule-1; sPECAM-1, soluble platelet and endothelial cell adhesion molecule-1; MMP-9, matrix metalloproteinase-9; TIMP-1, tissue inhibitor metalloproteinase-1.

$(7,9,11)$. Interestingly, in newborns with suspected EOS, specifically in the absence of these comorbidities, we showed that serum levels of the angiopoietins clearly discriminated newborns with bacteremia from those without bacteremia and from healthy controls (11).

With respect to sCAMs and sheddases, levels were not associated with bacteremia in suspected EOS in an earlier study in Surinamese newborns (12), and are not associated with bacteremia and mortality in LONS in the current study, which contrasts with results in children and adults $(5,15,16)$. The degree of endothelial cell activation is possibly reflected by circulating levels of its markers (5). A review by our group summarized that the degree of sCAM shedding may be different in newborns (5). Physiological events such as birth may drive high levels and further increases in the postnatal period, which 
reduce discriminatory and predictive properties in LONS during the neonatal period. For example, levels of sICAM increase in healthy newborns from days up to weeks after birth $(5,20,21)$, and were shown to be a marker of neonatal sepsis only in the first 4 days after birth (22). Time course follow-up studies of levels of markers of endothelial cell activation, strictly performed in a large cohort of newborns with suspected LONS and a control group, could give more insight into temporal aspects of endothelial physiology in the neonatal period.

It is tempting to speculate that endothelial physiology in (premature) newborns is different from relatively matured endothelium in children and adults, and responds differently to inflammatory stimuli. This premise is supported by experimental in vivo data. For example, in the study by Pierce et al., younger children without or with less severe sepsis had higher levels of Ang-1 and higher levels Ang-2 in case of severe sepsis (17). Furthermore, absolute levels of Ang-1 and Ang-2 are even higher in newborns with EOS as shown in our earlier study (11) and with LONS in the current study, indicating decreasing basal levels with increasing age. Interestingly, $\mathrm{sE}$-selectin release was found to be higher in septic than in healthy newborns in clinical studies, but the effect was less pronounced than in children and adults $(5,21,23-26)$. Thus, the endothelium of the newborn may be developing and have a variable ability to respond to inflammatory stimuli, driving different levels of its markers. Studies in neonatal mice with sepsis may help to study the effect of (premature) age on the response pattern of endothelial cells in sepsis in both organs and blood.

Important limitations of this study are the relatively small sample size and the fact that no further dilution of serum was possible to retrieve sVCAM-1 levels. Also, outlier data points exceeding 2,500 $\mathrm{ng} / \mathrm{mL}$, may have skewed the sE-selectin levels, since earlier studies report levels $<1,500 \mathrm{ng} / \mathrm{mL}$. Last, the use of serum may have caused release of MMP-9, TIMP-1 and NE from granules of neutrophils during the clotting process, which may have led to higher levels. Strength of this study is the use of blood culture as golden standard. Furthermore, our study provides much-needed data on neonatal sepsis in developing countries like Suriname, where infection rates remain high (13).

In conclusion, markers of endothelial cell activation are poorly associated with bacteremia and mortality in suspected LONS in this study. This contrasts with observations in children and adults, and is probably due to age-related (patho)physiological discrepancies. In addition, comorbidities and interventions during their stay at the neonatal care facility may drive non-discriminatory levels of these markers, thereby limiting their clinical utility in suspected LONS. Although only larger studies will be able to rule out all potential associations between these markers and clinical outcomes definitely, our results indicate that the clinical value of these markers is limited.

\section{Acknowledgments}

The authors acknowledge the efforts of all employees of the Clinical Laboratory of the Academic Hospital Paramaribo and the Central Laboratory of Suriname, Paramaribo, Suriname, for assistance with sample storage, handling, and transport.

Funding: This work was supported by the Thrasher Research Fund (grant number TRF13064) (R Zonneveld) and Tergooi Hospitals, Blaricum, The Netherlands.

\section{Footnote}

Conflicts of Interest: The authors have no conflicts of interest to declare.

Etbical Statement: The authors are accountable for all aspects of the work in ensuring that questions related to the accuracy or integrity of any part of the work are appropriately investigated and resolved. The Surinamese Medical-Ethical Board approved the study (VG-021-14A) and an amendment (VG-021-14A-2015_2016) for inclusion of newborns with a GA below 32 weeks and/or birth weight below 1,500 grams. Written informed consent for the use of serum and clinical information was obtained from at least one parent.

\section{References}

1. Aird WC. The role of the endothelium in severe sepsis and multiple organ dysfunction syndrome. Blood 2003;101:3765-77.

2. Fiedler U, Augustin HG. Angiopoietins: a link between angiogenesis and inflammation. Trends Immunol 2006;27:552-8.

3. Leligdowicz A, Richard-Greenblatt $M$, Wright J, et al. Endothelial Activation: The Ang/Tie Axis in Sepsis. Front Immunol 2018;9:838.

4. Kümpers $P$, van Meurs M, David S, et al. Time course of angiopoietin-2 release during experimental human 
endotoxemia and sepsis. Crit Care 2009;13:R64.

5. Zonneveld R, Martinelli R, Shapiro NI, et al. Soluble adhesion molecules as markers for sepsis and the potential pathophysiological discrepancy in neonates, children and adults. Crit Care 2014;18:204.

6. Paulus P, Carla Jennewein, Zacharowski K. Biomarkers of endothelial dysfunction: can they help us deciphering systemic inflammation and sepsis? Biomarkers 2011;16:S11-21.

7. Fang Y, Li C, Shao R, et al. Prognostic significance of the angiopoietin-2/angiopoietin-1 and angiopoietin-1/Tie2 ratios for early sepsis in an emergency department. Crit Care 2015;19:367.

8. Giuliano JS, Lahni PM, Harmon K, et al. Admission angiopoietin levels in children with septic shock. Shock 2007;28:650-4.

9. Wright JK, Hayford K, Tran V, et al. Biomarkers of endothelial dysfunction predict sepsis mortality in young infants: a matched case-control study. BMC Pediatr 2018;18:118.

10. Sharma D, Farahbakhsh N, Shastri S, et al. Biomarkers for diagnosis of neonatal sepsis: a literature review. J Matern Fetal Neonatal Med 2018;31:1646-59.

11. Zonneveld R, Jongman R, Juliana A, et al. Low Serum Angiopoietin-1, High Serum Angiopoietin-2, and High Ang-2/Ang-1 Protein Ratio are Associated with Early Onset Sepsis in Surinamese Newborns. Shock 2017;48:638-43.

12. Zonneveld R, Jongman RM, Juliana A, et al. Serum concentrations of endothelial cell adhesion molecules and their shedding enzymes and early onset sepsis in newborns in Suriname. BMJ Paediatr Open 2018;2:e000312.

13. Zonneveld R, Holband N, Bertolini A, et al. Improved referral and survival of newborns after scaling up of intensive care in Suriname. BMC Pediatr 2017;17:189.

14. Xing K, Murthy S, Liles WC, et al. Clinical utility of biomarkers of endothelial activation in sepsis-a systematic review. Crit Care 2012;16:R7.

15. de Pablo R, Monserrat J, Reyes E, et al. Circulating sICAM-1 and sE-Selectin as biomarker of infection and prognosis in patients with systemic inflammatory response syndrome. Eur J Intern Med 2013;24:132-8.

16. Skibsted S, Jones AE, Puskarich MA, et al. Biomarkers of endothelial cell activation in early sepsis. Shock 2013;39:427-32.

17. Pierce RW, Shabanova V, Canarie M, et al. Angiopoietin
Level Trajectories in Toddlers With Severe Sepsis and Septic Shock and Their Effect on Capillary Endothelium. Shock 2019;51:298-305.

18. Chan KY, Leung FW, Lam HS, et al. Immunoregulatory protein profiles of necrotizing enterocolitis versus spontaneous intestinal perforation in preterm infants. PLoS One 2012;7:e36977.

19. Maliba R, Brkovic A, Neagoe PE, et al. Angiopoietin-mediated endothelial P-selectin translocation: cell signaling mechanisms. J Leukoc Biol 2008;83:352-60.

20. Phocas I, Sarandakou A, Giannaki G, et al. Soluble intercellular adhesion molecule-1 in newborn infants. Eur J Pediatr 1998;157:153-6.

21. Giannaki G, Rizos D, Xyni K, et al. Serum soluble E- and L-selectin in the very early neonatal period. Early Hum Dev 2000;60:149-55.

22. Hansen AB, Vender H, Staun-Olsen P. Soluble intercellular adhesion molecule and C-reactive protein as early markers of infection in newborns. J Perinat Med 2000;28:97-103.

23. Døllner H, Vatten L, Austgulen R. Early diagnostic markers for neonatal sepsis: comparing C-reactive protein, interleukin-6, soluble tumour necrosis factor receptors and soluble adhesion molecules. J Clin Epidemiol 2001;54:1251-7.

24. Austgulen R, Arntzen KJ, Hæreid PE, et al. Infections in neonates delivered at term are associated with increased serum levels of ICAM-1 and E-selectin. Acta Paediatr 1997;86:274-80.

25. Edgar JDM, Gabriel V, Gallimore JR, et al. A prospective study of the sensitivity, specificity and diagnostic performance of soluble intercellular adhesion molecule 1, highly sensitive C-reactive protein, soluble E-selectin and serum amyloid $\mathrm{A}$ in the diagnosis of neonatal infection. BMC Pediatr 2010;10:22.

26. Edgar JDM, Wilson DC, McMillan SA, et al. Predictive Value of Soluble Immunological Mediators in Neonatal Infection. Clin Sci 1994;87:165-71.

Cite this article as: Achten NB, van Meurs M, Jongman RM, Juliana A, Molema G, Plötz FB, Zonneveld R. Markers of endothelial cell activation in suspected late onset neonatal sepsis in Surinamese newborns: a pilot study. Transl Pediatr 2019;8(5):412-418. doi: $10.21037 /$ tp.2019.11.03 